\title{
Case Report \\ Posterior Reversible Leukoencephalopathy Syndrome in a Patient after Acute COVID-19 Infection
}

\author{
Ketino Kobaidze $\left(\mathbb{D},{ }^{1}\right.$ Yoo Mee Shin $\left(\mathbb{D},{ }^{2}\right.$ Mariam Japaridze $\left(\mathbb{D},{ }^{3}\right.$ Ioannis Karakis $\left(\mathbb{D},{ }^{4}\right.$ \\ and $X i n$ Wu ${ }^{5}$ \\ ${ }^{1}$ Associate Professor of Medicine, Emory University School of Medicine, Division of Hospital Medicine 550 Peachtree Street, \\ Atlanta, GA 30308, USA \\ ${ }^{2}$ Division of Hospital Medicine, Emory University School of Medicine, Atlanta, GA 30308, USA \\ ${ }^{3}$ Ross University School of Medicine, New Jersey, USA \\ ${ }^{4}$ Department of Neurology, Emory University School of Medicine, Atlanta, GA 30308, USA \\ ${ }^{5}$ Department of Radiology and Imaging Science, Emory University School of Medicine, Atlanta, GA 30308, USA
}

Correspondence should be addressed to Ketino Kobaidze; kkobaid@emory.edu

Received 25 January 2021; Revised 30 March 2021; Accepted 15 April 2021; Published 27 April 2021

Academic Editor: Isabella Laura Simone

Copyright (C) 2021 Ketino Kobaidze et al. This is an open access article distributed under the Creative Commons Attribution License, which permits unrestricted use, distribution, and reproduction in any medium, provided the original work is properly cited.

The SARS-CoV-2 infection affects numerous organs, including the central nervous system. The neuroinvasive abilities and neuroinflammation may lead to short- and long-term neurological manifestations. Among neurological disorders associated with SARS-CoV-2 infection, posterior reversible encephalopathy syndrome (PRES) has been described in a few case-based observational studies during the acute phase of COVID-19 hospitalization. We present a case of a patient who developed seizures and PRES after recovering from an acute severe COVID-19 infection.

A 90-year-old African American female with multiple comorbidities and a severe COVID-19 infection was discharged home in stable condition after two weeks of hospitalization. A week later, she developed new-onset generalized tonic-clonic seizures requiring readmission to the hospital. The patient's clinical course and brain imaging supported PRES. Her mentation returned to baseline with supportive care and anticonvulsant treatment. Follow-up brain MRI four months later demonstrated resolution of FLAIR signal abnormalities confirming PRES. SARS-CoV-2 insult on the cerebrovascular endothelial cells likely continued and despite the clinical recovery eventually resulted in PRES. We believe that this is the first case describing the presentation of PRES after recovery from severe acute COVID-19 infection.

\section{Introduction}

Posterior reversible encephalopathy (PRES) is a neurological syndrome of cerebral dysautoregulation which can occur in multiple settings, including but not limited to acute migraine, occipital epilepsy, eclampsia/preeclampsia, hypertensive encephalopathy, and the use of cytotoxic and immunosuppressive medications [1]. While helpful in establishing the diagnosis, neuroimaging studies are unable to distinguish between the varying etiologies. Disruption of cerebral autoregulation combined with endothelial dysfunction appears to play important roles in the development of this clinical condition, which has been seen to be a result of transitional vasospasm, ischemic injury, or vasogenic edema, due to increased capillary permeability. Patients in all age groups are susceptible, though it has a slightly higher predilection for women.

The currently ongoing novel COVID-19 pandemic has affected millions of individuals across the globe, and there are over 539, 038 fatal cases reported in the United States alone [2]. Widespread dysregulation of pulmonary, renal, cardiac, and circulatory homeostasis contributes to morbidity and mortality in COVID-19 patients [3]. Knowledge about COVID-19's clinical presentation and disease 
progression is rapidly growing. However, less known are longer-term outcomes, especially for patients who are discharged from hospitals after severe acute COVID-19 infection.

There is already ample evidence that neurological complications of SARS-CoV-2 are common and may precede respiratory symptoms [4-8]. The expression of angiotensin-converting enzyme 2 (ACE-2) in brain vasculature creates an opportunity for the virus to enter into CNS cells, resulting in harm to neurological tissue $[6,9,10]$. It alters the blood-brain barrier permeability, which may result in brain autoregulation and vascular circulation disruption [11]. Several neurological complications of COVID-19 infection have been described in the literature. Patients may present with headache, acute encephalopathy, encephalitis, ischemic or hemorrhagic stroke, demyelinating neuropathy (Guillain-Barre syndrome), anosmia, ageusia, and seizures $[3,6,7,9]$.

Endothelial dysfunction in the setting of COVID-19 may contribute to PRES, as this complication has been described among hospitalized patients with COVID-19 [5]. Neurologic effects of SARS-CoV-2 may have long-term consequences. We present a case of an elderly patient who developed seizures and PRES after recovering from the acute phase of COVID-19 infection. We believe that this rare complication was associated with the recent SAR-CoV-2 virus infection affecting the nervous system.

\section{Case Presentation}

A 90-year-old African American female with a history of type 2 diabetes, essential hypertension, deep venous thrombosis, pulmonary embolism, and atrial flutter on chronic anticoagulation with apixaban presented for newonset general tonic-clonic seizures, witnessed by her family. The patient had been bed-bound from arthritis in a nursing home, but her mental status had been intact. The patient had recently been hospitalized over 3 weeks ago for COVID-19 pneumonia and was discharged home with home hospice one week before readmission.

According to her family, the patient's mental status had severely declined during her previous hospitalization with COVID-19. She developed staring spells after discharge that culminated into generalized tonic-clonic seizures on the day of the current admission. The episode lasted two seconds. Per her hospice nurse, blood pressures and blood glucose were well controlled at home. The home medications were thoroughly reviewed and found not to have any typical pharmacologic culprits. When she presented to the emergency department, she had two more witnessed seizures. Her blood pressure during this episode was 227/95 mm Hg, her heart rate was 98 beats $/ \mathrm{min}$, the temperature was $36.7^{\circ} \mathrm{C}$, her respiratory rate was 16 breaths/min, and oxygen saturation was $93 \%$ while she breathed room air. Intravenous labetalol, lorazepam, and levetiracetam were administered. Her blood pressure decreased to $160 / 70 \mathrm{~mm} \mathrm{Hg}$ and remained well controlled during her hospitalization. Head CT and basic laboratory work (Table 1) were unremarkable. Her physical exam was notable for postictal confusion. She was alert and only oriented to person. An electroencephalogram (EEG) detected no evidence of seizure or epileptiform discharges, but generalized slowing with an intermittent focal slowing in the bilateral temporal regions was noticed (Table 1). Brain MRI demonstrated subcortical and cortical FLAIR signal abnormality involving the left greater than right parietooccipital lobes and the left temporal lobe, in a pattern most compatible with posterior reversible encephalopathy syndrome (PRES) (Figures 1(a)-1(d)). There was no acute intracranial hemorrhage or infarction.

The patient had no further seizures after being treated with levetiracetam. Her mental state gradually returned to normal. She became more eager to participate in physical therapy and be more independent. She set a goal to walk without her walker and to cook for her family and friends. Her family was pleasantly surprised by this improvement in her mentation. She had no further seizure activity in the hospital and was discharged back home with home care services. She is doing well six months after discharge, is seizure-free, and follows her scheduled appointments. Follow-up MRI four months later after presentation showed complete or near resolution of the lesions (Figures 1(e)-1(h)).

No written consent has been obtained from the patient as there is no patient identifiable data included in the case report.

\section{Discussion}

The pathogenesis of PRES is controversial and not always clearly defined [10-15]. We speculate that the COVID-19 virus-associated endothelial dysfunction progressed after the acute phase of the disease and likely contributed to PRES in our patient.

PRES has been described in COVID-19 patients in a few case-based observational studies set during the acute phase of COVID-19 hospitalization $[1,16,17]$. We believe that this is the first case describing the presentation of PRES after discharge from initial COVID-19 hospitalization, which may reflect a delayed neurological sequela of SARS-CoV-2 requiring readmission. Our patient did not receive any specific medications for COVID-19 infection but was treated in a clinical trial known as the RESCUE trial with chest single low-dose radiation therapy for severe pneumonia [18].

Our patient's blood pressure during her presentation was elevated only on one occasion after grand mal seizure but was well controlled throughout both hospitalizations and at home. A list of home medications was thoroughly reviewed and found not to have any typical pharmacologic culprits. Her recovery from severe COVID-19 infectionassociated pneumonia, acute renal failure, and seizure from PRES was remarkable given her age and multiple comorbidities. Treatment of PRES depends on identifying any potential triggers and removing them. Our patient was treated with supportive care and investigational single low-dose chest radiation during her initial hospitalization [19]. We guess that PRES could be a potential complication of severe COVID-19 infection and in appropriate clinical settings, and PRES diagnosis may prompt the clinician to consider a diagnosis of COVID-19 in the acute or reconvalescent phase. 
TABLE 1: Clinical characteristics during initial hospitalization and readmission.

\begin{tabular}{|c|c|c|}
\hline Variables & Initial admission & Readmission \\
\hline Reason for admission & Fever & Seizure \\
\hline Comorbidities & $\begin{array}{l}\text { HTN, osteoarthritis, DM 2, VTE, CKD stage 2, atrial } \\
\text { flutter, cataract, macular degeneration, right eye } \\
\text { blindness, pressure ulcer stage II, mild dementia }\end{array}$ & $\begin{array}{l}\text { HTN, osteoarthritis, DM 2, VTE, CKD stage 2, atrial } \\
\text { flutter, cataract, macular degeneration, right eye } \\
\text { blindness, pressure ulcer stage II, mild dementia }\end{array}$ \\
\hline COVID-19 PCR & $\begin{array}{c}\text { Positive } \\
\text { At discharge: negative }\end{array}$ & Not tested \\
\hline Symptoms of COVID-19 & $\begin{array}{c}\text { Fever and cough, acute, respiratory failure, altered } \\
\text { mental status }\end{array}$ & NA \\
\hline Hospital problem list & $\begin{array}{l}\text { Acute respiratory failure, viral pneumonia, acute } \\
\text { diffuse body pain, DM 2, HTN, acute renal failure, } \\
\text { delirium, cystitis, hypokalemia }\end{array}$ & $\begin{array}{l}\text { New-onset seizure, acute metabolic encephalopathy, } \\
\text { cystitis, hypomagnesemia, hypokalemia }\end{array}$ \\
\hline Risk factors for PRES & N/A & BP fluctuations, recent COVID-19 infection \\
\hline $\begin{array}{l}\text { The onset of PRES from } \\
\text { diagnosing COVID-19 }\end{array}$ & NA & 31 days \\
\hline LOS, total & 22 days & 6 days \\
\hline LOS, ICU & 2 days & 0 \\
\hline LOS, floor & 20 days & 6 days \\
\hline MAP, range & $81-120$ & $91-113$ \\
\hline SBP, range & $124-192$ & $127-227$ \\
\hline Medications & $\begin{array}{l}\text { Acetaminophen, apixaban, ceftriaxone, insulin, } \\
\text { labetalol, ondansetron, magnesium sulfate, potassium } \\
\text { chloride, hydralazine, gabapentin, tramadol }\end{array}$ & $\begin{array}{l}\text { Acetaminophen, apixaban, labetalol, ceftriaxone, } \\
\text { insulin, levetiracetam, tramadol (stopped at admission) }\end{array}$ \\
\hline
\end{tabular}

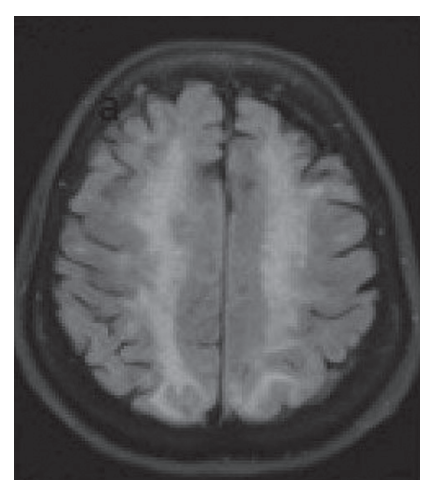

(a)

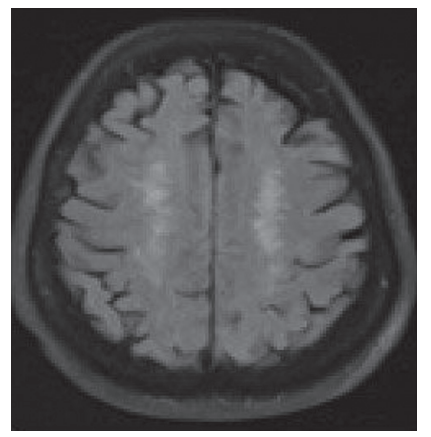

(e)

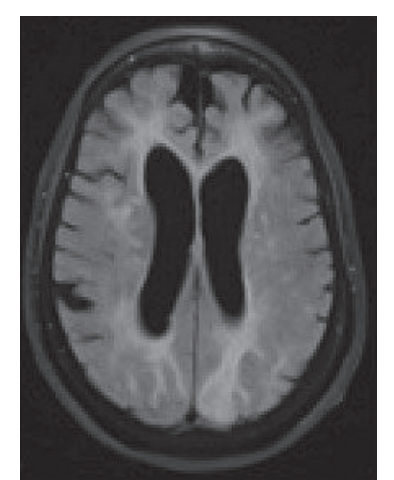

(b)

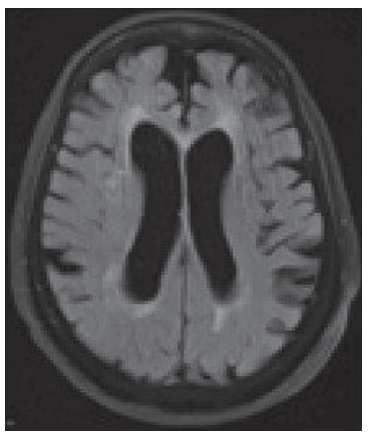

(f)

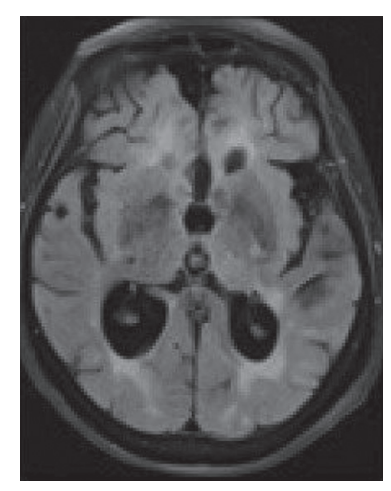

(c)

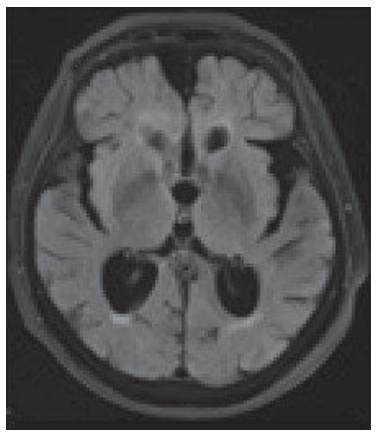

(g)

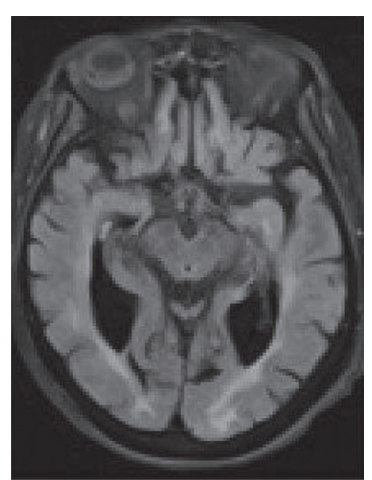

(d)

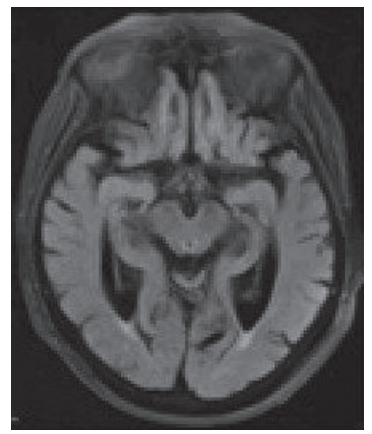

(h)

FIgURE 1: (a-d) Axial FLAIR images at multiple levels demonstrate cortical, subcortical, and periventricular signal hyperintensity and mild mass effect typical of PRES, predominantly involving the bilateral frontal, parietal, and occipital lobes on admission. Follow-up MRI (e-h) images four months after presentation showed resolution of the lesions. 


\section{Conflicts of Interest}

The authors declare that they have no conflicts of interest.

\section{References}

[1] M. Fischer and E. Schmutzhard, "Posterior reversible encephalopathy syndrome," Journal of Neurology, vol. 264, no. 8, pp. 1608-1616, 2017.

[2] http://www.cdc.gov/covid-data-tracker/\#cases.

[3] H. Li, S.-M. Liu, X.-H. Yu, S.-L. Tang, and C.-K. Tang, "Coronavirus disease 2019 (COVID-19): current status and future perspectives," International Journal of Antimicrobial Agents, vol. 55, no. 5, p. 105951, 2020.

[4] A. Whittaker, M. Anson, and A. Harky, "Neurological Manifestations of COVID-19: a systematic review and current update," Acta Neurologica Scandinavica, vol. 142, no. 1, pp. 14-22, 2020.

[5] L. Kishfy, M. Casasola, P. Banankhah et al., "Posterior reversible encephalopathy syndrome (PRES) as a neurological association in severe Covid-19," Journal of the Neurological Sciences, vol. 414, p. 116943, 2020.

[6] Y. Kaya, S. Kara, C. Akinci, and A. S. Kocaman, "Transient cortical blindness in COVID-19 pneumonia; a PRES-like syndrome: case report," Journal of the Neurological Sciences, vol. 413, p. 116858, 2020.

[7] R. Bridwell, B. Long, and M. Gottlieb, "Neurologic complications of COVID-19," American Journal of Emergency Medicine, vol. 38, no. 7, p. 1549, 2020.

[8] A. M. Baig, A. Khaleeq, U. Ali, and H. Syeda, "Evidence of the COVID-19 virus targeting the CNS: tissue distribution, hostvirus interaction, and proposed neurotropic mechanisms," ACS Chemical Neuroscience, vol. 11, no. 7, pp. 995-998, 2020.

[9] I. Ahmad and F. A. Rathore, "Neurological manifestations and complications of COVID-19: a literature review," Journal of Clinical Neuroscience, vol. 77, pp. 8-12, 2020.

[10] A. Agarwal, M. Pinho, K. Raj et al., "Neurological emergencies associated with COVID-19: stroke and beyond," Emergency Radiology, vol. 27, no. 6, p. 747, 2020.

[11] T. Coolen, V. Lolli, N. Sadeghi et al., "Early postmortem brain MRI findings in COVID-19 non-survivors," Neurology, vol. 95, pp. e2016-e2027, 2020.

[12] I. Alquisiras-Burgos, I. Peralta-Arrieta, L. A. Alonso-Palomares, A. E. Zacapala-Gómez, E. G. Salmerón-Bárcenas, and P. Aguilera, "Neurological complications associated with the blood-brain barrier damage induced by the inflammatory response during SARS-CoV-2 infection," Molecular Neurobiology, vol. 58, no. 2, pp. 520-535, 2021.

[13] R. Garg, N. Kumar, and H. Malhotra, "Posterior reversible encephalopathy syndrome in eclampsia," Neurology India, vol. 66, no. 5, pp. 1316-1323, 2018.

[14] S. Deguchi, K. Mitsuya, Y. Nakasu et al., "Posterior reversible encephalopathy syndrome (PRES) induced by pazopanib, a multi-targeting tyrosine kinase inhibitor, in a patient with soft-tissue sarcoma: case report and review of the literature," Investigational New Drugs, vol. 36, no. 2, p. 346, 2018.

[15] J. Shankar and J. Banfield, "Posterior reversible encephalopathy syndrome: a review," Canadian Association of $\mathrm{Ra}$ diologists Journal, vol. 68, no. 2, pp. 147-153, 2017.

[16] F. X. Doo, G. Kassim, D. R. Lefton, S. Patterson, H. Pham, and P. Belani, "Rare presentations of COVID19 PRES-like leukoencephalopathy and carotid thrombosis," Clinical Imaging, vol. 69, pp. 94-101, 2020.
[17] L. Princiotta Cariddi, P. Tabaee Damavandi, F. Carimati et al., "Reversible Encephalopathy Syndrome (PRES) in a COVID19 patient," Journal of Neurology, vol. 267, pp. 3157-3160, 2020.

[18] S. C. Parauda, V. Gao, A. N. Gewirtz et al., "Posterior reversible encephalopathy syndrome in patients with COVID19," Journal of the Neurological Sciences, vol. 416, p. 117019, 2020 Jul 9.

[19] C. B. Hess, Z. S. Buchwald, W. Stokes et al., "Low-dose wholelung radiation for COVID-19 pneumonia: planned day 7 interim analysis of a registered clinical trial," Cancer, vol. 126, no. 23, pp. 5109-5113, 2020. 